TRANSACTIONS OF THE

AMERICAN MATHEMATICAL SOCIETY

Volume 211, 1975

\title{
RESULTS ON SUMS OF CONTINUED FRACTIONS
}

BY

\author{
JAMES L. HLAVKA
}

ABSTRACT. Let $F(m)$ be the (Cantor) set of infinite continued fractions with partial quotients no greater than $m$ and let $F(m)+F(n)=$ $\{a+\beta: a \in F(m), \beta \in F(n)\}$. We show that $F(3)+F(4)$ is an interval of length $1.14 \ldots$ so every real number is the sum of an integer, an element of $F(3)$ and an element of $F(4)$. Similar results are given for $F(2)+F(7), F(2)+F(2)+F(4), F(2)+F(3)+F(3)$ and $F(2)+F(2)+$ $F(2)+F(2)$. The techniques used are applicable to any Cantor sets in $R$ for which certain parameters can be evaluated.

Marshall Hall, Jr. [3] proved that $F(4)+F(4) \equiv \mathbf{R}(\bmod 1)$ (all notation is defined in the next paragraph) and posed the question: is $F(3)+F(4) \equiv \mathbf{R}$ $(\bmod 1)$ ? In this paper we prove $F(3)+F(4) \equiv \mathbf{R}(\bmod 1)$ and several other results, summarized in Table 1 . Only two questions concerning when a sum of $F\left(m_{i}\right) \equiv \mathbf{R}$ remain open: $F(2)+F(5) \equiv \mathbf{R}$ ? and $F(2)+F(6) \equiv \mathbf{R}$ ? We conjecture that they are both false. '

$$
\begin{array}{llll}
F(2)+F(4) \equiv \mathbf{R} & F(3)+F(3) \equiv \mathbf{R} & F(2)+F(2)+F(3) \equiv \mathbf{R} \\
F(2)+F(5) ? & F(3)+F(4) \equiv \mathbf{R} & F(2)+F(2)+F(4) \equiv \mathbf{R} \\
F(2)+F(6) ? & & F(2)+F(3)+F(3) \equiv \mathbf{R} \\
F(2)+F(7) \equiv \mathbf{R} & & F(2)+F(2)+F(2)+F(2) \equiv \mathbf{R}
\end{array}
$$

Table 1. All congruences are modulo 1

We let $\mathbf{N}$ be the natural numbers and $\mathbf{R}$ the real numbers. Lower case Roman letters except $g$ and $h$ will be- elements of $\mathbf{N}$; Greek letters elements of R. Let

$$
\left\langle a_{1}, a_{2}, \ldots\right\rangle=\frac{1}{a_{1}}+\frac{1}{a_{2}}+\ldots,
$$

Received by the editors June 25,1974 .

AMS (MOS) subject classifications (1970). Primary 10A30, 10F20; Secondary $10 \mathrm{~F} 40,10 \mathrm{~J} 99$.

Key words and phrases. Continued fractions, sums of Cantor sets, congruence modulo 1. 


$$
\begin{aligned}
& \left\langle a_{1}, \ldots, a_{r}, \overline{a_{r+1}, \ldots, a_{s}}\right\rangle \\
& \quad=\left\langle a_{1}, \ldots, a_{r}, a_{r+1}, \ldots, a_{s}, a_{r+1}, \ldots, a_{s}, \ldots\right\rangle,
\end{aligned}
$$

and

$$
F(m)=\left\{\left\langle a_{1}, a_{2}, \ldots\right\rangle: 1 \leq a_{i} \leq m \text { for all } i \in \mathbf{N}\right\} .
$$

When working with continued fractions it is convenient to write intervals without ordering their endpoints, so we define

$$
(\alpha, \beta)=\{\xi \in \mathbf{R}: \min (\alpha, \beta)<\xi<\max (\alpha, \beta)\}
$$

and

$$
[\alpha, \beta]=\{\xi \in \mathbf{R}: \min (\alpha, \beta) \leq \xi \leq \max (\alpha, \beta)\} .
$$

If $A$ and $B$ are subsets of $R$, let $\bar{A}=$ the span of $A=\sup (\alpha-\beta)$ over all $\alpha, \beta \in A$ and $A+B=\{\alpha+\beta: \alpha \in A, \beta \in B\}$. Write " $A+B \equiv \mathbf{R}(\bmod 1) "$ to mean " $\xi \in \mathbf{R}$ implies $\xi \equiv \alpha+\beta(\bmod 1)$ for some $\alpha \in A, \beta \in B$." Let $P(m)$ be the special closed interval $[\langle\overline{m, 1}\rangle, \overline{1, m}\rangle]$.

Note that $\langle\overline{m, 1}\rangle$ and $\langle\overline{1, m}\rangle$ are the least and greatest elements of $F(m)$, respectively, so $F(m) \subset P(m)$. Moreover $F(m) \subset F(m+1)$. The latter inclusion immediately shows that every question of the form $\Sigma_{i} F\left(m_{i}\right) \equiv \mathbf{R}$ ? is covered in Table 1.

$F(m)$ is a Cantor set so the natural approach to computing $\Sigma F\left(m_{i}\right)$ is by deleting intervals from the $P\left(m_{i}\right)$. Our objective is to devise an algorithm, called a construction of $F(m)$, which controls the order of these deletions sufficiently to establish that $\Sigma F\left(m_{i}\right)=\Sigma P\left(m_{i}\right)$ whenever this is true. (This is the same approach Hall used to investigate $F(4)+F(4)$.)

Lemma 1. $F(m)=P(m) \backslash O(m)=$ the set-theoretic difference of $P(m)$ and $O(m)$, where $O(m)$ is defined by.

$$
\begin{array}{r}
O(m)=\bigcup\left\{\left(\left\langle a_{1}, \ldots, a_{s}, \overline{1, m\rangle},\left\langle a_{1}, \ldots, a_{s}+1, \overline{m, 1}\right\rangle\right): s \in N,\right.\right. \\
\left.1 \leq a_{i} \leq m \text { for } i \leq s \text { and } a_{s} \neq m\right\} .
\end{array}
$$

Proof. We show that $O(m)$ is composed of precisely those intervals which are deleted from $P(m)$ to form $F(m)$.

First assume $a \in P(m) \backslash F(m)$. Then $a$ has a first partial quotient $a_{r+1}$ which is greater than $m$, with $r>0$ and $a_{r} \neq 1$ when $r=1$. Now if $a_{r}=1$ then

$$
\begin{aligned}
& a=\left\langle a_{1}, \ldots, a_{r-1}, a_{r}, \ldots\right\rangle \\
& \quad \in\left(\left\langle a_{1}, \ldots, a_{r-1}+1, \overline{m, 1}\right\rangle,\left\langle a_{1}, \ldots, a_{r-1}, \overline{1, m}\right\rangle\right)
\end{aligned}
$$


and if $a_{r}>1$ then

$$
a \in\left(\left\langle a_{1}, \ldots, a_{r}-1, \overline{1, m}\right\rangle,\left\langle a_{1}, \ldots, a_{r}, \overline{m, 1}\right\rangle\right),
$$

so $a \in O(m)$. Conversely if $a \in\left(\left\langle a_{1}, \ldots, a_{s}, \overline{1, m}\right\rangle,\left\langle a_{1}, \ldots, a_{s}+1\right.\right.$, $\overline{m, 1}\rangle) \subset O(m)$, then

(2) $a \in\left(\left\langle a_{1}, \ldots, a_{s}, \overline{1, m}\right\rangle,\left\langle a_{1}, \ldots, a_{s}+1\right\rangle\right)$,

(3) $a=\left\langle a_{1}, \ldots, a_{s}+1\right\rangle$, or

(4) $a \in\left(\left\langle a_{1}, \ldots, a_{s}+1\right\rangle,\left\langle a_{1}, \ldots, a_{s}+1, \overline{m, 1}\right\rangle\right)$.

If (2) then $\alpha=\left\langle a_{1}, \ldots, a_{s}, \alpha^{\prime}\right\rangle$ where $\langle\overline{1, m}\rangle\left\langle\alpha^{\prime}<1\right.$ so $\alpha^{\prime} \& F(m)$. Hence $\alpha \notin F(m)$. If (4) then $a=\left\langle a_{1}, \ldots, a_{s+1}, \alpha^{\prime}\right\rangle$ where $\alpha^{\prime}\langle\langle\overline{m, 1}\rangle$ and again $a \notin F(m)$. Lastly, (3) implies $a \notin F(m)$ since $F(m)$ contains only infinite continued fractions.

We now define a construction of $F(m)$ as follows. Let $I_{m}^{2}=P(m)$. Choose an interval $O_{m}^{2}$ of $O(m)$ and delete it from $I_{m}^{2}$, leaving two new intervals $I_{m}^{3}$ and $I_{m}^{4}$. From each delete an interval of $O(m)$, say $O_{m}^{3}$ and $O_{m}^{4}$ respectively. $I_{m}^{3}$ will be split into two intervals $I_{m}^{5}$ and $I_{m}^{6} ; I_{m}^{4}$ will be split into $I_{m}^{7}$ and $I_{m}^{8}$. From each of $I_{m}^{5}, \ldots, I_{m}^{8}$ delete the interval $O_{m}^{5}, \ldots, O_{m}^{8}$ respectively. Continue in this way. This procedure is demonstrated in Figures $1 \mathrm{a}$ and $1 \mathrm{~b}$. If $O(m)=\bigcup_{i=2}^{\infty} O_{m}^{i}$ we call this procedure a construction $C$ of $F(m)$. We call $F_{m}^{k}=\bigcup_{j=2^{k-1}+1}^{2^{k}} p_{m}^{j}$ the $k$ th step in the construction of $F(m)$.

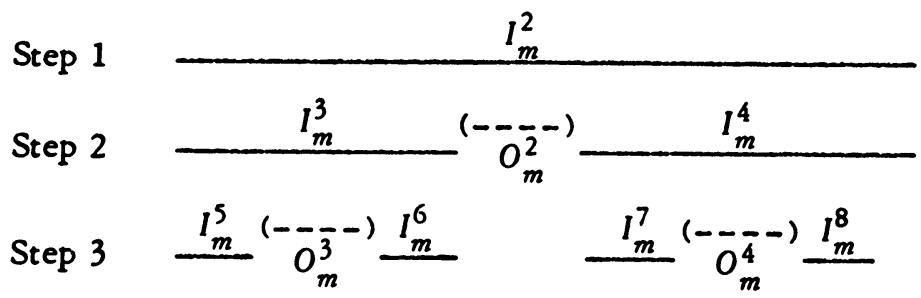

Figure la

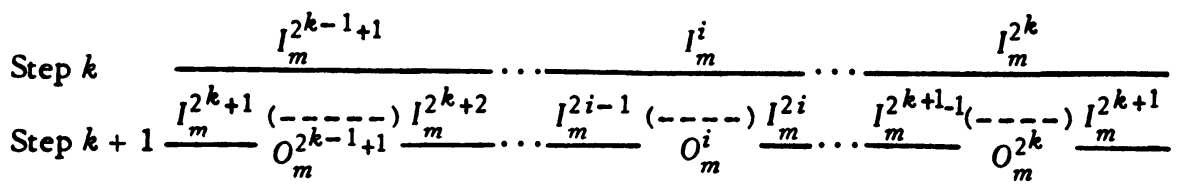

Figure lb 
Lemma 2. If $C$ is any construction of $F(m), m>1$, and $F_{m}^{k}$ is the kth step in this construction, then $F(m)=\bigcap_{k=1}^{\infty} F_{m}^{k}$.

Proof. Obvious from Figure 1b.

Definition. If $C$ is a construction of $F(m)$, then

$$
\begin{gathered}
g_{m}=g_{m}(C)=\sup _{i}\left(\overline{O_{m}^{i}} / \overline{I_{m}^{i}}\right), \\
h_{m}=h_{m}(C)=\inf \left(\inf _{i}\left(\overline{I_{m}^{2 i-1}} / \overline{I_{m}^{i}}\right), \inf _{i}\left(\overline{I_{m}^{2 i}} / I_{m}^{i}\right)\right),
\end{gathered}
$$

and

$$
h_{m}^{\prime}=h_{m}^{\prime}(C)=\sup \left(\sup _{i}\left(\overline{l_{m}^{2 i-1}} / \overline{l_{m}^{i}}\right), \sup _{i}\left(\overline{I_{m}^{2 i}} / \bar{l}_{m}^{\bar{i}}\right)\right)
$$

Theorem 3. If there exist constructions $C$ and $C^{\prime}$ of $F(m)$ and $F(n)$ respectively such that

(5) $g_{m}(C) \cdot g_{n}\left(C^{\prime}\right) \leq h_{m}(C) \cdot h_{n}\left(C^{\prime}\right)$ and

(6) $g_{m}(C) \cdot \overline{P(m)} \leq \overline{P(n)}$ and $g_{n}\left(C^{\prime}\right) \cdot \overline{P(n)} \leq \overline{P(m)}$

then $F(m)+F(n)=P(m)+P(n)$.

Proof. Let $\left\{I_{m}^{i}\right\}_{i=2}^{\infty}$ and $\left\{I_{n}^{j}\right\}_{j=2}^{\infty}$ be the intervals appearing in the constructions $C$ and $C^{\prime}$ respectively. Call the intervals $I_{m}^{i}$ and $I_{n}^{j}$ compatible (with respect to $C$ and $C^{\prime}$ ), written $I_{m}^{i} \sim I_{n}^{j}$, iff

$$
\mathrm{g}_{m} \cdot \overline{l_{m}^{i}} \leq \overline{l_{n}^{j}} \text { and } g_{n} \cdot \overline{l_{n}^{j}} \leq \overline{l_{m}^{i}} \text {. }
$$

Call the intervals $I_{m}^{i}$ and $I_{n}^{j} M$-dividable, written $I_{m}^{i} \underset{\sim}{\sim} I_{n}^{j}$, iff (8) or (9) is true, where (8) and (9) are the following (symmetric) conditions.

$$
l_{m}^{2 i-1} \sim l_{n}^{j} \text { and } l_{m}^{2 i} \sim p_{n}^{j},
$$

(8.2) $\left(I_{m}^{2 i-1}+P_{n}^{j}\right) \cup\left(I_{m}^{2 i}+p_{n}^{j}\right)=I_{m}^{i}+p_{n}^{j}, \quad$ and

$$
M \cdot \overline{l_{m}^{i}} \geq \overline{l_{n}^{j}}
$$

(9)

$$
I_{m}^{i} \sim I_{n}^{2 j-1} \text { and } I_{m}^{i} \sim I_{n}^{2 j}
$$

(9.2) $\left(I_{m}^{i}+I_{n}^{2 j-1}\right) \cup\left(I_{m}^{i}+I_{n}^{2 j}\right)=l_{m}^{i}+l_{n}^{j}$, and

$$
M \cdot \overline{p_{n}^{j}} \geq \overline{i_{m}^{i}} \text {. }
$$


The four pairs of intervals appearing in (8.1) and (9.1) are said to be derived from the pair $\left(I_{m}^{i}, l_{n}^{j}\right)$.

It suffices to show that for some $M \in \mathbf{R}^{+}, I_{m}^{i} \sim l_{n}^{j}$ implies $I_{m}^{i} \stackrel{M}{\approx} l_{n}^{j}$ for all $i, j \geq 2$. To prove this, set $S_{0}=\left\{\left(I_{m}^{2}, I_{n}^{2}\right)\right\}$ and

$S_{r+1}=\left\{(I, J): I \sim J\right.$ and $(I, J)$ is derived from a pair $\left.\left(I_{0}, J_{0}\right) \in S_{r}\right\}$.

Clearly

$\bigcup\left\{I+J:(I, J) \in S_{r+1}\right\}=\bigcup\left\{I+J:(I, J) \in S_{r}\right\}=\cdots=I_{m}^{2}+I_{n}^{2}=P(m)+P(n)$.

If $(I, J) \in S_{r}$ then $\bar{I} \cdot \bar{J} \leq \lambda^{r} \cdot \overline{I_{m}^{2}} \cdot \overline{l_{n}^{2}} \rightarrow 0$ as $r \rightarrow \infty$, where $\lambda=$ $\max \left(1-b_{m}, 1-b_{n}\right)<1$ (if $h_{m}$ or $h_{n}=0$ then $g_{m}$ or $g_{n}=0$ by (5) so $F(m)$ or $F(n)$ is not a Cantor set-contradiction). Since $I \sim J$, the ratio $\bar{I} / \bar{J}$ is bounded so $\bar{I} \rightarrow 0$ and $\bar{J} \rightarrow 0$. Therefore for each $i$ there is an $r_{0}$ such that $O_{m}^{i}$ has been deleted from every $l$ appearing in a pair $(I, J) \in S_{r}, r>r_{0^{*}}$ Since $O(m)=\bigcup_{i=2}^{\infty} O_{m}^{i}$,

$$
\alpha(m) \cap\left(\bigcap_{r=0}^{\infty}\left(U\left\{I:(I, J) \in S_{r}\right\}\right)\right)=\varnothing .
$$

But for all $r, F(m) \subset \bigcup\left\{l:(I, J) \in S_{r}\right\} \subset P(m)$ so (10) and Lemma 1 yield

$$
F(m)=\bigcap_{r=0}^{\infty}\left(\bigcup\left\{I:(I, J) \in S_{r}\right\}\right)
$$

Similarly for $F(n)$. Since the sequence $\left\{U\left\{I:(I, J) \in S_{r}\right\}\right\}_{r=0}^{\infty}$ is a nested sequence of compact sets, we obtain directly the result

$$
F(m)+F(n)=\bigcap_{r=0}^{\infty}\left(\bigcup\left\{I+J:(I, J) \in S_{r}\right\}\right)=\bigcap_{r=0}^{\infty}(P(m)+P(n))=P(m)+P(n) .
$$

Now fix $M \geq \max \left(h_{m} / g_{n}, h_{n} / g_{m}\right)$ and assume $l_{m}^{i} \sim l_{n}^{j}$. Since $g_{m} g_{n} \leq$ $b_{m} h_{n}$, we must have

$$
\overline{I_{m}^{i}} / \overline{I_{n}^{j}} \geq g_{n} / h_{m}
$$

or

$$
\overline{I_{m}^{i}} / \overline{l_{n}^{j}} \leq h_{n} / g_{m}
$$

Assuming (11) we will verify (8). Similarly (9) will follow from (12), so this will show $I_{m}^{i} \stackrel{M}{\approx} I_{n^{j}}^{j}$. So assume (11) and set $k=2 i-1$ or $2 i$. Then recalling the definition of $b_{m}$, 


$$
\overline{l_{m}^{k}} \geq h_{m} \cdot \overline{I_{m}^{i}} \geq g_{n} \cdot \overline{i_{n}^{j}} \text { and } \overline{p_{n}^{j}} \geq g_{m} \cdot \overline{l_{m}^{i}} \geq g_{m} \cdot \overline{l_{m}^{k}} \text {, }
$$

so $l_{m}^{k} \sim l_{n}^{j}$. To check (8.2), let $I_{m}^{2 i-1}=[\alpha, \beta], l_{m}^{2 i}=[y, \delta]$ and $l_{n}^{j}=\left[a_{0}\right.$, $\delta_{0}$, with $\alpha<\beta<\gamma<\delta$ and $\alpha_{0}<\delta_{0}$. Since

$$
\overline{p_{n}^{j}} \geq g_{m} \cdot \overline{l_{m}^{i}} \geq \overline{O_{m}^{i}}
$$

we have $\delta_{0}-a_{0} \geq \gamma-\beta$ or $\beta+\delta_{0} \geq \gamma+a_{0}$. Then

$$
\begin{aligned}
\left(l_{m}^{2 i-1}+p_{n}^{j}\right) \cup\left(l_{m}^{2 i}+l_{n}^{j}\right) & =\left[\alpha+a_{0}, \beta+\delta_{0}\right] \cup\left[\gamma+a_{0}, \delta+\delta_{0}\right] \\
& =\left[a+a_{0}, \delta+\delta_{0}\right]=l_{m}^{i}+l_{n}^{j} .
\end{aligned}
$$

Lastly, (8.3) is satisfied by our choice of $M$.

Lemma 4. If $\left.\left.\alpha=\left\langle a_{1}, \ldots, a_{s}, \alpha^{\prime}\right\rangle, \beta=\left\langle a_{1}, \ldots, a_{s}, \beta^{\prime}\right\rangle, \alpha^{\prime}\right\rangle 0, \beta^{\prime}\right\rangle 0$, $\left\langle a_{1}, \ldots, a_{s}\right\rangle=p_{s} / q_{s},\left\langle a_{1}, \ldots, a_{s-1}\right\rangle=p_{s-1} / q_{s-1}$ and $Q=q_{s-1} / q_{s}$, then

$$
\left(\alpha^{\prime}-\beta^{\prime}\right) /(\alpha-\beta)=\left(Q+\alpha^{\prime}\right)\left(Q+\beta^{\prime}\right)(-1)^{s+1} q_{s}^{2} .
$$

Proof. We have

$$
\begin{aligned}
\alpha-\beta & =\frac{p_{s} \alpha^{\prime}+p_{s-1}}{q_{s} a^{\prime}+q_{s-1}}-\frac{p_{s} \beta^{\prime}+p_{s-1}}{q_{s} \beta^{\prime}+q_{s-1}} \\
& =\frac{p_{s}}{q_{s}}\left\{\frac{\alpha^{\prime}+Q+p_{s-1} / p_{s}-Q}{\alpha^{\prime}+q_{s-1} / q_{s}}-\frac{\beta^{\prime}+Q+p_{s-1} / p_{s}-Q}{\beta^{\prime}+q_{s-1} / q_{s}}\right\} \\
& =\frac{p_{s}}{q_{s}}\left(\frac{p_{s-1}}{p_{s}}-Q\right)\left\{\frac{1}{\alpha^{\prime}+Q}-\frac{1}{\beta^{\prime}+Q}\right\} \\
& =\frac{(-1)^{s+1}\left(\alpha^{\prime}-\beta^{\prime}\right)}{q_{s}^{2}\left(\alpha^{\prime}+Q\right)\left(\beta^{\prime}+Q\right)},
\end{aligned}
$$

since $p_{s-1} q_{s}-p_{s} q_{s-1}=(-1)^{s}$. The result follows immediately.

Lemma 5. If $\left.\left.\gamma=\left\langle a_{1}, \ldots, a_{s}, \gamma^{\prime}\right\rangle, \delta=\left\langle a_{1}, \ldots, a_{s}, \delta^{\prime}\right\rangle, \gamma^{\prime}\right\rangle 0, \delta^{\prime}\right\rangle 0$, and $\alpha, \beta, \alpha^{\prime}, \beta^{\prime}, Q$ are as in Lemma 4 , then

$$
\frac{\alpha-\beta}{\gamma-\delta}=\frac{a^{\prime}-\beta^{\prime}}{\gamma^{\prime}-\delta^{\prime}} \cdot \frac{\left(Q+\gamma^{\prime}\right)\left(Q+\delta^{\prime}\right)}{\left(Q+a^{\prime}\right)\left(Q+\beta^{\prime}\right)}
$$

and $Q \in\left[1 /\left(a_{s}+1\right), 1\right]$.

Proof. Statement (14) is an immediate corollary of Lemma 4. The restriction on $Q$ follows from the well-known result that $Q=\left\langle a_{s}, \ldots, a_{1}\right\rangle$. 
Theorem 6. $F(3)+F(4)=P(3)+P(4)=[\langle\overline{3,1}\rangle+\langle\overline{4,1}\rangle,\langle\overline{1,3}\rangle+\langle\overline{1,4}\rangle]=$ [. $4709 \ldots, 1.6197 \ldots .$.$] .$

Proof. We produce constructions of $F(3)$ and $F(4)$ satisfying the hypotheses of Theorem 3. Let us begin by defining a canonical construction, $C_{m}$, of $F(m)$ for any $m$, as follows. $I_{m}^{2}$ must be $[\overline{m, 1}, \overline{1, m})$. If

$$
l_{m}^{i}=\left[\left\langle a_{1}, \ldots, a_{s}, j, \overline{m, 1}\right\rangle,\left\langle a_{1}, \ldots, a_{s}, m, \overline{1, m}\right\rangle\right]
$$

with $s \geq 0$ and $j \neq m$ then

$$
O_{m}^{i}=\left(\left\langle a_{1}, \ldots, a_{s}, j, \overline{1, m}\right\rangle,\left\langle a_{1}, \ldots, a_{s}, j+1, \overline{m, 1}\right\rangle\right)
$$

so that

and

$$
I_{m}^{2 i-1}=\left[\left\langle a_{1}, \ldots, a_{s}, j, \overline{m, 1}\right\rangle,\left\langle a_{1}, \ldots, a_{s}, j, \overline{1, m}\right\rangle\right]
$$

$$
I_{m}^{2 i}=\left[\left\langle a_{1}, \ldots, a_{s}, j+1, \overline{m, 1}\right\rangle,\left\langle a_{1}, \ldots, a_{s}, m, \overline{1, m}\right\rangle\right]
$$

It is relatively easy to show that $\bigcup_{i=2}^{\infty} O_{m}^{i}=O(m)$ so this does define a construction of $F(m)$. The value of the constructions $C_{m}$ is that we can readily calculate $g_{m}\left(C_{m}\right)$ and $h_{m}\left(C_{m}\right)$ (and $h_{m}^{\prime}\left(C_{m}\right)$, which will be needed later). We have

$$
\begin{aligned}
g_{m}\left(C_{m}\right) & =\max _{i \geq 2}\left(\overline{O_{m}^{i}} / \overline{I_{m}^{i}}\right) \\
& =\max \frac{\left\langle a_{1}, \ldots, a_{s}, j, \overline{1, m}\right\rangle-\left\langle a_{1}, \ldots, a_{s}, j+1, \overline{m, 1}\right\rangle}{\left\langle a_{1}, \ldots, a_{s}, j, \overline{m, 1}\right\rangle-\left\langle a_{1}, \ldots, a_{s}, m, \overline{1, m}\right\rangle}
\end{aligned}
$$

over $1 \leq j \leq m, s \geq 0$ and $1 \leq a_{i} \leq m$ for $i \leq s$. Using Lemma 5 , we obtain

$$
\boldsymbol{g}_{m}\left(C_{m}\right) \leq \max \left\{\frac{\langle j, \overline{1, m}\rangle-\langle j+1, \overline{m, 1}\rangle}{\langle j, \overline{m, 1}\rangle-\langle m, \overline{1, m}\rangle} \cdot \frac{(Q+\langle j, \overline{m, 1}\rangle)(Q+\langle m, \overline{1, m}))}{(Q+\langle j, \overline{1, m}\rangle)(Q+\langle j+1, \overline{m, 1}\rangle)}\right\}
$$

over $1 \leq j \leq m$ and $Q \in[1 /(m+1), 1]$. For each allowable value of $j$ this expression is a rational function in $Q$ whose maximum on the interval $[1 /(m+1), 1]$ can be readily calculated. $\Lambda$ Univac 1108 was used to perform these calculations and then maximize over $j$ (and for similar calculations arising later). We thus obtain the bounds

$$
g_{3}\left(C_{3}\right) \leq .2992 \ldots \quad \text { and } g_{4}\left(C_{4}\right) \leq .2278 \ldots
$$

Similarly 


$$
\begin{aligned}
& h_{m}\left(C_{m}\right)=\min _{i \geq 2}\left(\min \left(\overline{I_{m}^{2 i-1} / I_{m}^{i}}, \overline{I_{m}^{2 i} / I_{m}^{i}}\right)\right) \\
& =\min _{j, a_{i}}\left(\operatorname { m i n } \left(\frac{\left\langle a_{1}, \ldots, a_{s}, j, \overline{1, m}\right\rangle-\left\langle a_{1}, \ldots, a_{s}, j, \overline{m, 1}\right\rangle}{\left\langle a_{1}, \ldots, a_{s}, m, \overline{1, m}\right\rangle-\left\langle a_{1}, \ldots, a_{s}, j, \overline{m, 1}\right\rangle}\right.\right. \\
& \left.\left.\frac{\left\langle a_{1}, \ldots, a_{s}, m, \overline{1, m}\right\rangle-\left\langle a_{1}, \ldots, a_{s}, j+1, \overline{m, l}\right\rangle}{\left\langle a_{1}, \ldots, a_{s}, m, \overline{1, m}\right\rangle-\left\langle a_{1}, \ldots, a_{s}, j, \overline{\left.m_{0} 1\right\rangle}\right\rangle}\right)\right) \\
& \geq \min _{j, \boldsymbol{Q}}\left(\operatorname { m i n } \left(\frac{\langle j, \overline{1, m}\rangle-\langle j, \overline{m, 1}\rangle}{\langle m, \overline{1, m}\rangle-\langle j, \overline{m, 1}\rangle} \cdot \frac{(Q+\langle m, \overline{1, m}\rangle)}{(Q+\langle j, \overline{1, m}\rangle)},\right.\right. \\
& \left.\left.\frac{\langle m, \overline{1, m}\rangle-\langle j+1, \overline{m, 1}\rangle}{\langle m, \overline{1, m}\rangle-\langle j, \overline{m, 1}\rangle} \cdot \frac{(Q+\langle j, \overline{m, 1}\rangle)}{(Q+\langle j+1, \overline{m, 1}\rangle)}\right)\right),
\end{aligned}
$$

from which we obtain

$$
h_{3}\left(C_{3}\right) \geq .2471 \ldots \text { and } h_{4}\left(C_{4}\right) \geq .2963 \ldots \text {. }
$$

A simple multiplication shows that

$$
g_{3}\left(C_{3}\right) \cdot g_{4}\left(C_{4}\right) \leq .0667<.0731 \leq h_{3}\left(C_{3}\right) \cdot h_{4}\left(C_{4}\right) .
$$

Also

$$
g_{3} \cdot \overline{P(3)} \leq .2992 \times .5276<P(4)=.6212 \ldots<\frac{.5276}{.2278} \leq \frac{\overline{P(3)}}{g_{4}}
$$

so by Theorem 3 we have the result $F(3)+F(4)=P(3)+P(4)$.

Corollary 7. $F(3)+F(4) \equiv \mathbf{R}(\bmod 1)$.

Proof. This is obvious since $F(3)+F(4)$ contains an interval of length greater than one.

The values of $g_{3}, g_{4}, h_{3}$ and $h_{4}$ are in fact equal to the bounds given because these bounds arise from $Q=1 /(m+1)$ or $Q=1$, which are possible values of $Q$. For $g_{2}$ and $h_{2}$, below, this does not happen.

Applying Theorem 3 to the canonical constructions of $F(2)$ and $F(12)$ as above we can establish $F(2)+F(12)=P(2)+P(12)$, but now we find that the canonical construction of $F(12)$ is not an optimal construction in terms of minimizing the ratio $g_{12} / h_{12}$. This is because the maximal value of $\overline{O_{m}^{i}} / \overline{i_{m}^{i}}$ always occurs at $j=m-1$ but the minimal value of $\min \left(\overline{l_{m}^{2 i-1}}, \overline{\left.l_{m}^{2 i}\right) / \overline{l_{m}^{i}}}\right.$ 
only occurs at $j=m-1$.if $m \leq 4$. A noncanonical construction can allow us to lower the number 12 , but the best result is obtained by extending Theorem 3.

Theorem 8. If there exist constructions $C$ and $C^{\prime}$ of $F(m)$ and $F(n)$ respectively, such that

$$
\begin{aligned}
& \overline{O_{m}^{i}} \cdot \overline{O_{n}^{j}} \leq \min \left(\overline{I_{m}^{2 i-1}}, \overline{I_{m}^{2 i}}\right) \cdot \min \left(\overline{I_{n}^{2 j-1}}, \overline{\left.I_{n}^{2 j}\right)} \text { for all } i, j \geq 2\right. \text {, } \\
& g_{m} \cdot \overline{P(m)} \leq \overline{P(n)} \text { and } g_{n} \cdot \overline{P(n)} \leq \overline{P(m)} \text {, }
\end{aligned}
$$

and

$$
\max \left(h_{m}^{\prime}, h_{n}^{\prime}\right) \cdot g_{m} g_{n} \leq h_{m} h_{n}
$$

then $F(m)+F(n)=P(m)+P(n)$.

Proof. Call the intervals $I_{m}^{i}$ and $l_{n}^{j} M$-dividable ${ }^{*}$ iff (8), (9) or

$$
\begin{aligned}
\left(I_{m}^{2 i-1}+I_{n}^{2 j-1}\right) \cup\left(I_{m}^{2 i-1}+I_{n}^{2 j}\right) & \cup\left(I_{m}^{2 i}+I_{n}^{2 j-1}\right) \\
& \cup\left(I_{m}^{2 i}+I_{n}^{2 j}\right)=I_{m}^{i}+l_{n}^{j} \text { and }
\end{aligned}
$$

(18.2) $I_{m}^{2 i-1} \sim I_{n}^{2 j-1}, I_{m}^{2 i-1} \sim I_{n}^{2 j}, I_{m}^{2 i} \sim I_{n}^{2 j-1}$, and $I_{m}^{2 i} \sim I_{n}^{2 j}$

holds. The proof now parallels the proof of Theorem 3; the only significant difference being to show that a compatible pair is M-dividable* when neither (11) nor (12) holds. So assume

$$
h_{n} / g_{m} \leq \overline{I_{m}^{i}} / \overline{l_{n}^{j}} \leq g_{n} / h_{m}
$$

Let $l_{m}^{2 i-1}=[\alpha, \beta], I_{m}^{2 i}=[y, \delta], I_{n}^{2 j-1}=\left[\alpha_{0}, \beta_{0}\right]$, and $I_{n}^{2 j}=\left[\gamma_{0}, \delta_{0}\right]$. From (15) we obtain

$$
\overline{O_{m}^{i}} \leq \min \left(\overline{I_{n}^{2 j-1}}, \overline{\left.I_{n}^{2 j}\right)} \text { or } \overline{O_{n}^{j}} \leq \min \left(\overline{I_{m}^{2 i-1}}, \overline{\left.I_{m}^{2 i}\right)}\right.\right.
$$

assume for simplicity the latter. Then $\gamma_{0}-\beta_{0} \leq \min (\beta-\alpha, \delta-\gamma)$ so $\alpha+$ $\gamma_{0} \leq \beta+\beta_{0}$ and $\gamma+\gamma_{0} \leq \delta+\beta_{0}$. Then the LHS of (18.1) is

$$
\begin{gathered}
{\left[\alpha+a_{0}, \beta+\beta_{0}\right] \cup\left[\alpha+\gamma_{0}, \beta+\delta_{0}\right] \cup\left[\gamma+\alpha_{0}, \delta+\beta_{0}\right] \cup\left[\gamma+\gamma_{0}, \delta+\delta_{0}\right]} \\
=\left[\alpha+a_{0}, \beta+\delta_{0}\right] \cup\left[\gamma+\alpha_{0}, \delta+\delta_{0}\right]=\left[\alpha+\alpha_{0}, \delta+\delta_{0}\right]=I_{m}^{i}+p_{n}^{j}
\end{gathered}
$$

For $k=2 i-1$ or $2 i, l=2 j-1$ or $2 j$, we have

$$
\overline{l_{m}^{k}} \geq h_{m} \cdot \overline{l_{m}^{i}} \geq \frac{h_{m} h_{n}}{g_{m}} \cdot \overline{l_{n}^{j}} \geq \frac{h_{m} h_{n}}{g_{m} h_{n}^{\prime}} \cdot \bar{l}_{n}^{l} \geq g_{n} \cdot \overline{l_{n}^{l}}
$$


and similarly $\bar{I}_{n}^{\bar{l}} \geq g_{m} \cdot \bar{I}_{m}^{k}$ so $\overline{I_{m}^{k}} \sim \overline{I_{n}^{l}}$.

Theorem 9. $F(2)+F(7)=P(2)+P(7)=[.4928 . \ldots, 1.6195 \ldots ..] \equiv \mathbf{R}$ $(\bmod 1)$.

Proof. The canonical constructions can be used. We again apply Lemma 5 to obtain the bounds

$$
\begin{aligned}
& g_{2} \leq .4456 \ldots, h_{2} \geq .1686 \ldots, \quad h_{2}^{\prime} \leq .4589 . ., g_{7} \leq .1343 . ., \\
& h_{7} \geq .2906 \ldots, \quad h_{7}^{\prime} \leq .6359 . ., \quad \text { and } \frac{\overline{0_{7}^{j}}}{\min \left(\overline{l_{7}^{2 j-1}}, \overline{\left.l_{2}^{2 j}\right)}\right.} \leq .3594 \ldots .
\end{aligned}
$$

Since $\min \left(\overline{I_{2}^{2 i-1}}, \overline{I_{2}^{2 i}}\right) / \overline{O_{2}^{i}} \geq h_{2} / g_{2}$, it is now easy to verify the hypotheses of Theorem 8.

For any constructions of $F(2)$ and $F(6)$, equation (15) fails whenever an interval of the type $\left(\left\langle a_{1}, \ldots, a_{s}, 5, \overline{1,6\rangle},\left\langle a_{1}, \ldots, a_{s}, 6, \overline{6,1}\right\rangle\right)\right.$ is deleted from $F(6)$. Thus, as (15) is intuitively a "best possible" condition in the sense that no weakening approximations were made, it is probable that $F(2)+F(6) \neq P(2)+P(6)$. Moreover, since (15) fails infinitely often, $F(2)+F(6)$ may possibly be a Cantor set.

The negative results $F(3)+F(3) \equiv \mathbf{R}(\bmod 1)$ and $F(2)+F(4) \equiv \mathbf{R}$ (mod 1) can be verified directly. $P(2)+P(4)$ has length less than one and $F(3) \subset[\langle\overline{3,1}\rangle,\langle 3, \overline{3,1}\rangle] \cup[\langle 2, \overline{1,3}\rangle, \overline{1,3}\rangle]$ yields

$$
F(3)+F(3) \subset[.5274 \ldots, .62178 \ldots . .] \cup[.62200 \ldots, 1.5826 \ldots] \text {. }
$$

We now look at sums of $F\left(m_{1}\right)+\ldots+F\left(m_{s}\right)$ with $s>2$.

Theorem 10. Let $C_{1}, \ldots, C_{s}$ be constructions of $F\left(m_{1}\right), \ldots, F\left(m_{s}\right)$ respectively (not necessarily canonical constructions). If

(20) $h_{m} \leq \overline{I_{m}^{2}} / \overline{I_{n}^{2}}$ for $m, n \in\left\{m_{1}, \ldots, m_{s}\right\}$, and

(21) $g_{m}+h_{m} \leq \Sigma_{i=1}^{s} h_{m_{i}}$ for $m \in\left\{m_{1}, \ldots, m_{s}\right\}$ then $F\left(m_{1}\right)+\ldots+F\left(m_{s}\right)=P\left(m_{1}\right)+\ldots+P\left(m_{s}\right)$.

Proof. Again we mimic the proof of Theorem 3. Let $\left(I_{m_{1}}^{j}, \ldots, I_{m_{s}}^{j_{s}}\right)$ be compatible iff

$$
h_{m_{i}} \cdot \overline{i_{m_{k}}^{j_{k}}} \leq \overline{l_{m_{i}}^{j_{i}}} \text { for } 1 \leq i, k \leq s
$$


Call $\left(I_{m_{1}}^{j_{1}}, \ldots, I_{m_{s}}^{j_{s}}\right)$ dividable iff

$$
\left(I_{n}^{2 j-1}+\left(l_{m_{1}}^{j_{1}}+\cdots+{\widehat{p_{n}^{j}}}_{n}+\cdots+l_{m_{s}}^{j_{s}}\right)\right) \cup\left(I_{n}^{2 j}+\left(l_{m_{1}}^{j_{1}}+\cdots+\widehat{l}_{n}^{j}+\cdots+I_{m_{s}}^{j_{s}}\right)\right)
$$

$$
=l_{m_{1}}^{j_{1}}+\cdots+r_{m_{s}}^{j_{s}}
$$

and $\left(I_{m_{1}}^{j_{1}}, \ldots, I_{n}^{2 j-1}, \ldots, I_{m_{s}}^{j_{s}}\right)$ and $\left(I_{m_{1}}^{j_{1}}, \ldots, I_{n}^{2 j}, \ldots, I_{m_{s}}^{j_{s}}\right)$ are compatible, where means omission and $n$ is such that

$$
\overline{l_{n}^{j}} \geq \overline{l_{m_{i}}^{j_{i}}} \text { for } 1 \leq i \leq s .
$$

Hypothesis (20) says the beginning s-tuple $\left(I_{m_{1}}^{2}, \ldots, I_{m_{s}}^{2}\right)$ is compatible, so the proof reduces to showing that every compatible s-tuple is dividable. Let $k=2 j-1$ or $2 j$. Then $\overline{l_{n}^{k}} \geq h_{n} \cdot \overline{l_{n}^{j}} \geq h_{n} \cdot \overline{I_{m_{i}}^{i}}$ for $1 \leq i \leq s$, and the other combinations of subscripts occurring in (22) trivially produce correct inequalities, so $\left(I_{m_{1}}^{j}, \ldots, I_{n}^{k}, \ldots, I_{m_{s}}^{j}\right)$ is compatible. From (21), we have

$$
g_{n} \leq h_{m_{1}}+\cdots+\widehat{h}_{n}+\cdots+h_{m_{s}}
$$

so that

$$
\begin{aligned}
\overline{l_{m_{1}}^{j_{1}}}+\cdots+\widehat{\widehat{l_{n}^{j}}}+\cdots+ & +\overline{l_{m_{s}}^{j_{s}}} \geq h_{m_{1}} \cdot \overline{\bar{l}_{n}^{j}}+\cdots+{\widehat{h_{n}} \cdot \overline{l_{n}^{j}}}+\cdots+h_{m_{s}} \cdot \overline{l_{n}^{j}} \\
& =\left(h_{m_{1}}+\cdots+\widehat{h}_{n}+\cdots+h_{m_{s}}\right) \cdot l_{n}^{j} \geq g_{n} \cdot \overline{l_{n}^{j}} \geq \overline{O_{n}^{j}}
\end{aligned}
$$

This is the analog of equation (13) and is precisely the inequality needed to establish (23).

Theorem 11. The following are all true.

$$
\begin{aligned}
& F(2)+F(2)+F(4)=P(2)+P(2)+P(4) \equiv \mathbf{R}(\bmod 1), \\
& F(2)+F(3)+F(3)=P(2)+P(3)+P(3) \equiv \mathbf{R}(\bmod 1),
\end{aligned}
$$

and

$$
F(2)+F(2)+F(2)+F(2)=P(2)+P(2)+P(2)+P(2) \equiv \mathbf{R}(\bmod 1)
$$

Proof. Apply Theorem 10 to the canonical constructions of $F(2), F(3)$ and $F(4)$.

The final result listed in Table $1, F(2)+F(2)+F(3) \equiv \mathbf{R}$, results from inspecting the first few subdivisions of $F(2)$ and $F(3)$. 
T. W. Cusick and R. A. Lee [1], [2] have investigated $\Sigma_{i} S\left(m_{i}\right)$, where

$$
\begin{aligned}
S(m)= & \left\{\left\langle a_{1}, a_{2}, \ldots\right\rangle: a_{i} \geq m \text { for all } i\right\} \\
& \cup\left\{\left\langle a_{1}, \ldots, a_{s}\right\rangle: a_{i} \geq m \text { for } 1 \leq i \leq s, s \geq 1\right\} \cup\{0\} .
\end{aligned}
$$

They have shown [2] that

$$
\sum_{i=1}^{m} S(m)=[0,1] \text {. }
$$

Our Theorem 10 can be applied to $\Sigma_{i} S\left(m_{i}\right)$ in place of $\Sigma_{i} F\left(m_{i}\right)$, whereupon (25) follows as a relatively easy special case.

More generally, Theorems 3, 8 and 10 are applicable to any Cantor sets for which $g_{m}, h_{m}$ and $h_{m}^{\prime}$ can be evaluated.

\section{BIBLIOGRAPHY}

1. T. W. Cusick, Sums and products of continued fractions, Proc. Amer. Math. Soc. 27 (1971), 35-38. MR 42 \#4498.

2. T. W. Cusick and R. A. Lee, Sums of sets of continued fractions, Proc. Amer. Math. Soc. 30 (1971), 241-246. MR 44 \#158.

3. Marshall Hall, Jro, On the sum and product of continued fractions, Ann. of Math. (2) 48 (1947), 966-993. MR 9, 226.

DEPARTMENT OF MATHEMATICS, UNIVERSITY OF WISCONSIN, MADISON, WISCONSIN 53705 